$\omega$ and therefore $N$ being prime to $p$. We have further

$$
\left[\frac{d^{p-1}(V X)}{d v}\right]_{v=0} \equiv X(1)\left[\frac{d^{p-1} V}{d v}\right]_{v=0} \equiv-X(1)(\bmod p) .
$$

Comparison of (5), (4) and (3) gives the theorem (1).

Philadelphia,

April, 1918.

\title{
TRAJECTORIES AND FLAT POINTS ON RULED SURFACES.
}

BY MR. J. K. WHITTEMORE.

(Read before the American Mathematical Society April 28 and October $27,1917$.

\$1. Introduction. In the following paper we determine the flat points* of a ruled surface with real rulings, and prove a new property of the orthogonal trajectories of the rulings. This property may be extended to any isogonal trajectory of the rulings, not itself a ruling, and may be regarded as a generalization of Bonnet's familiar theorem. $\dagger$

Let $S$ be a ruled surface with real rulings, $g$ any such ruling, $C$ an orthogonal trajectory of the rulings, generally not a straight line; let the coordinates of any point of $C$ be $x_{0}, y_{0}, z_{0}$, and consider these as functions of $v$, the arc of $C$. When $C$ is not a straight line let the direction cosines of its tangent be $\alpha, \beta, \gamma$, of its principal normal be $l, m, n$, and of its binormal be $\lambda, \mu, \nu$; let $R$ and $T$ be respectively the radii of curvature and torsion of $C, \psi$ the angle measured from the principal normal towards the binormal to the direction chosen as positive on $g$. We suppose $C$ to be a rectifiable curve generally without singular points in the portion considered; the curvature $1 / R$ and the torsion $1 / T$ shall have finite first derivatives with respect to $v$, and $\psi$ shall have a finite second derivative.

The surface $S$ is given by

$$
x=x_{0}+u L, \quad L=l \cos \psi+\lambda \sin \psi,
$$

* Flat points are defined in $\S 4$ of this paper.

$\dagger$ See Eisenhart, Differential Geometry, p. 248. 
with similar equations for $y$ and $z$, where $u$ is the length measured from $C$ on $g$ in the positive direction. If $C$ is a straight line $S$ is a right conoid. To give its equations we choose $C$ as the $X$ axis and let $\psi$ be the angle made by the positive direction on $g$ measured from the $Y$ axis towards the $Z$ axis. We have

$$
x=v, \quad y=u \cos \psi, \quad z=u \sin \psi .
$$

The linear element of (1) is given by

$$
\begin{aligned}
d s^{2} & =d u^{2}+\left[1-2 u q+u^{2}\left(p^{2}+q^{2}\right)\right] d v^{2} \\
p & =\frac{1}{T}-\psi^{\prime} \quad q=\frac{\cos \psi}{R} .
\end{aligned}
$$

For $\left(1^{\prime}\right) d s$ is also given by (2) making $1 / R=1 / T=0 . \quad S$ is developable if $p$ is identically zero; if $q$ is also identically zero $S$ is a plane or a cylinder.

It is well known* that the linear element of $S$ may be given the form

$$
d s^{2}=d u_{1}^{2}+\left[\left(u_{1}-a\right)^{2}+b^{2}\right] d v_{1}^{2},
$$

where the curves $\left(v_{1}\right)$, that is $v_{1}$ constant, are the rulings, $u_{1}$ is the length measured along a ruling from an orthogonal trajectory of the rulings, $u_{1}=0, a$ depending on $v_{1}$ alone the distance from this trajectory to the central point of the ruling so that $u_{1}=a$ is the equation of $s$, the line of striction of $S$; $b$ also depending on $v_{1}$ alone is the parameter of distribution of $S$. The total curvature $K$ of $S$ from (2) and (3) is

$$
K=\frac{-p^{2}}{\left[1-2 u q+u^{2}\left(p^{2}+q^{2}\right)\right]^{2}}=\frac{-b^{2}}{\left[\left(u_{1}-a\right)^{2}+b^{2}\right]^{2}} .
$$

Comparing (2) and (3), we have

$$
a=\frac{q}{p^{2}+q^{2}} \quad b=\frac{p}{p^{2}+q^{2}},
$$

the sign of $b$ being determined in the usual way. $\dagger$

$\S 2$. A property of the trajectories. Suppose a point $v$ of $C$ is on $s$; for this point $a=0$, and $q=\cos \psi / R=0$. If $1 / R=0$ for all points of $C$ that curve is a straight line, a

* Eisenhart, p. 247.

$\dagger$ Eisenhart, p. 246. 
geodesic, and by Bonnet's theorem is $s$; if $1 / R=0$ for the point $v$, but not for all points of $C$, it follows that $C$ is at this point tangent to the asymptotic line of $S$, not $g$, through $v$, unless the point $v$ is a flat point. If $1 / R \neq 0, \cos \psi=0$ and the normal to $S$ at $v$ coincides with the principal normal of $C$, and the geodesic curvature of $C$ vanishes at the point. If at a point of intersection of $C$ and $s$ the former is tangent to an asymptotic line its principal normal is not necessarily normal to $S$, as may be seen from the following construction: let $C$ be any curve satisfying the conditions of $\S 1$, $v$ a point of $C$ for which $1 / R=0,1 / T \neq 0$, let $S$ be a surface of normals to $C, \psi$ having any constant value. The point $v$ is on $s$ since $q=0, p=1 / T \neq 0 ; S$ has at this point an ordinary point which is not a flat point since $K \neq 0$. The curve $C$ is tangent to an asymptotic line at $v$, and the angle of the normal to $S$ and the principal normal of $C$ at $v$ is the complement of $\psi$.

Suppose conversely that $v$ is a point of $C$ where the geodesic curvature vanishes; then $\cos \psi=0, q=0$, and, unless $p=0$, $a=0$, and $v$ is on $s$. If $p=0, K=0$ for $v$ and consequently for all points of the ruling through $v$. Such a ruling we shall call a zero ruling. In the next section we examine the behavior of orthogonal trajectories of the rulings at points of intersection with zero rulings.

The properties of orthogonal trajectories just proved may be extended without modification to all isogonal trajectoriesa ruling itself not being considered, however, as a trajectory. We may state the results of this section in

Theorem 1: At a point of intersection of an isogonal trajectory of the rulings of a surface $S$ with the line of striction the geodesic curvature of the trajectory vanishes unless the tangent to the latter is asymptotic or the point is a flat point of $S$; conversely, a point of a trajectory where the geodesic curvature vanishes is either on the line of striction or on a zero ruling.

\$3. Trajectories on zero rulings. If the total curvature $K$ vanishes for $v$ and a finite value of $u, p=0$; either $b=0$, or $a$ or $b$ or both become infinite. If $q \neq 0, b=0$ and $a=1 / q$ is finite; if $q=0$ either $a$ or $b$ becomes infinite. We distinguish two cases of zero rulings: (I) $p=0, q \neq 0$; (II) $p=q=0$. That this is a distinction independent of the trajectory $C$ appears from the corresponding properties of $a$ and $b$, as also from properties of the point of the spherical indicatrix of $S$ corresponding to the ruling, which are stated below. 
Let $D, D^{\prime}, D^{\prime \prime}$ be the coefficients of the second fundamental form of $S ; D$ is identically zero, and $K=0$ gives $D^{\prime}=0$. The last condition gives

$$
\left|L L^{\prime} \alpha\right|=0 \quad L^{\prime}=-\alpha q+(l \sin \psi-\lambda \cos \psi) p .
$$

For (I)

For (II)

$$
\frac{\alpha}{L^{\prime}}=\frac{\beta}{M^{\prime}}=\frac{\gamma}{N^{\prime}}=-\frac{1}{q} .
$$

$$
L^{\prime}=M^{\prime}=N^{\prime}=0 .
$$

In (I) the point of the spherical indicatrix of $S$ corresponding to $v$, that is the point of coordinates $(L, M, N)$, is a simple point of the indicatrix, and the tangent to the indicatrix at $v$ is parallel to the tangent to $C$ at $v$. The orthogonal trajectory intersecting a zero ruling (I) on $s$ has at the point of intersection a singular point, as may be seen in various ways: (1) $a=0$ gives $R=0$; (2) the tangent to the spherical indicatrix at a point corresponding to a ruling $g$ is parallel to the normal to $S$ at the point of intersection of $g$ and $s,{ }^{*}$ but we have seen that the tangent to the indicatrix is parallel to the tangent to the orthogonal trajectory, a contradiction in the case of an ordinary point of the trajectory on $s$. This remark proves incidentally that the tangent to any other orthogonal trajectory at a point of intersection with a zero ruling (I) is parallel to the normal to $S$ at the intersection of the ruling with $s$. Since in (I) $\cos \psi \neq 0$ no orthogonal trajectory has zero geodesic curvature at a point on the ruling.

In (II) $q=0$ gives $\cos \psi=0$ or $1 / R=0$; if $\cos \psi=0$ the trajectory has zero geodesic curvature; if $1 / R=0$ the point is a flat point of $S$, for the ruling has the only asymptotic direction at a point for which $K=0$. The behavior of a trajectory at a flat point will be considered in the following section. To a zero ruling (II) corresponds a singular point of the spherical indicatrix since

$$
L^{\prime}=M^{\prime}=N^{\prime}=0 .
$$

An example of a zero ruling (II) is furnished by the surface of binormals of a curve $C$ at a point for which the torsion vanishes. In this example $b$ is infinite and $a$ may have any value.

* Eisenhart, p. 351. This does not apply to a developable surface. 
$\S 4$. Flat points. A flat point of a surface is a point where the curvature of every normal section is zero; it may also be defined as an umbilic where the total curvature of the surface vanishes.* The conditions for an umbilic are, in the usual notation,

$$
\frac{D}{E}=\frac{D^{\prime}}{F}=\frac{D^{\prime \prime}}{G} ;
$$

for a flat point $D=D^{\prime}=D^{\prime \prime}=0$. For $S$, with the coordinates $u, v$ used in the preceding sections, $D=0, E=1$, so that such a surface has no umbilics other than flat points. It is worth while at this point to emphasize the requirement that $S$ be a surface with real rulings, for there exists a real ruled surface with no flat points all of whose real points are umbilics-the sphere.

To determine the flat points of a surface $S$ we have, in addition to the identity $D=0$ and the condition for zero total curvature $D^{\prime}=0$, from which $v$ may be found, the equation for $u, D^{\prime \prime}=0$, which gives

(4) $u^{2}\left|L M^{\prime} N^{\prime \prime}\right|+u\left\{\left|x_{0}^{\prime \prime} M N^{\prime}\right|-\left|x_{0}{ }^{\prime} M N^{\prime \prime}\right|\right\}$

$$
+\left|x_{0}^{\prime \prime} M z_{0}^{\prime}\right|=0
$$

in which each bracket of three letters represents a determinant with principal diagonal in evidence.

For a zero ruling (I) equation (4) has two roots,

$$
\begin{gathered}
u=-\frac{1}{\sqrt{\Sigma L^{\prime 2}}}=\frac{1}{q} \quad u=-\frac{\left|x_{0}^{\prime \prime} M N^{\prime}\right|}{\left|L M^{\prime} N^{\prime \prime}\right|}=\frac{\sin \psi}{R t} \\
t=\frac{\sin \psi \cos \psi}{R^{2}}-p^{\prime} .
\end{gathered}
$$

The first value gives the central point of the ruling which is generally not a flat point but is a singular point of the coordinate curve $(u)$, the orthogonal trajectory through the point. The second value gives the same point if $p^{\prime}=0$, $\sin \psi \neq 0$; if $p^{\prime}=\sin \psi=0, D^{\prime \prime}=0$ for all $u$ 's, and all points of the ruling are flat points; if $p^{\prime} \neq 0, t \neq 0$, the general case, there is one flat point; if $p^{\prime} \neq 0, t=0$ there is no flat point.

For a zero ruling (II) the coefficient of $u^{2}$ in (4) vanishes, and that equation gives for a flat point one value,

* Scheffers, Theorie der Flächen, 2d ed., pp. 126, 146. 


$$
u=\frac{\mid x_{0}{ }^{\prime \prime} M z_{0}^{\prime}}{\left|x_{0}^{\prime} M N^{\prime \prime}\right|}=-\frac{\sin \psi}{R p^{\prime}} .
$$

The ruling has one finite flat point if $p^{\prime} \neq 0$; if $p^{\prime}=0$ there is no flat point unless also $1 / R=0$; if $p^{\prime}=1 / R=0$ all points of the ruling are flat points for $D^{\prime \prime}=0$ for all $u$ 's. The case of the right conoid, where $C$ is a straight line, is included in (II), for since $1 / R=0, q=0$; one flat point is on $C$, and all other or no other points of the ruling are flat points according as $p^{\prime}=-\psi^{\prime \prime}$ does or does not vanish.

In (I) we have $a \cos \psi=R$, so that the projection of the central point of the ruling on the osculating plane of any orthogonal trajectory $C$, satisfying the requirements of $\S 1$, is the center of curvature of the corresponding point of the trajectory. In the case of a single flat point in (I) suppose for the moment $C$ is the orthogonal trajectory through that point and that $C$ satisfies the requirements of $\S 1$; we have

$$
u=\frac{\sin \psi}{R t}=0 .
$$

Since $a$ is finite $1 / R \neq 0$, and $\sin \psi=0$, and the ruling is the principal normal of the trajectory through the flat point, and the central point of the ruling is the corresponding center of curvature of that trjaectory. If in (I) all points of the ruling are flat points $\sin \psi=0$ for any orthogonal trajectory satisfying the requirements of $\S 1$; the ruling is the principal normal and the central point the center of curvature of all such trajectories. The latter case is illustrated by taking for $S$ a plane regarded as the locus of tangents of one of its curves $s$. It may be proved if $p^{\prime}=0, \sin \psi \neq 0$, no flat point, that the principal normals of all orthogonal trajectories at points of the zero ruling are parallel. This case is illustrated on any ruling of a developable surface whose points are not flat points. In the case remaining in $(\mathrm{I}), p^{\prime} \neq 0, t=0$, no flat point, there is no simple relation between the ruling and the trajectories.

In (II) the ruling is the binormal of every orthogonal trajectory not intersecting the ruling at a flat point. If there is a single flat point on the ruling, $\psi$ may have any value at this point; if all points of the ruling are flat points there is no simple relation between the ruling and the trajectories.

By a discussion similar to the preceding we may prove analogous properties of isogonal-not orthogonal-trajectories of the rulings of $S$. We choose such a trajectory as the direc- 
trix $C$, and suppose $C$ cuts the rulings at the angle $A \neq 0$. No trajectory has zero geodesic curvature at a point of intersection with a zero ruling (I). Every trajectory through the central point of a ruling (I) has at this point a singular point, but if $A \neq \pi / 2$ the coordinate curve (u) through the central point has not a singular point at this point, so that the point is generally not a singular point of the surface. This case is again illustrated by a plane regarded as the locus of the tangents of one of its curves. Every trajectory intersecting a zero ruling (II) at a point not a flat point has zero geodesic curvature at this point.

We consider finally the behavior of the flat points when a surface $S$ is deformed into a surface of the same sort with correspondence of rulings.* From (2) it follows that in such deformation the values of $p^{2}$ and $q$ are unchanged for any point of $C$; therefore a zero ruling remains such and moreover remains always in the same case, (I) or (II). Since the arc $v$ is also unchanged the same is true of the absolute value of $p^{\prime}$, but $1 / R$ and $\psi$ are generally changed, so that the flat point moves on the ruling during deformation. In (I) deformation cannot change a ruling of one flat point into a ruling of all flat points, but can change either of these into a ruling with no flat point. In (II) a ruling of one flat point remains such during deformation, but a ruling of no flat points may be changed into a ruling of all flat points. A developable surface never has a ruling with a single flat point. The deformations for rulings of all and no flat points are illustrated for (I) by a plane regarded as the locus of tangents of one of its curves, for (II) by a cylinder. If all points of a surface are flat points the surface is a plane. $\dagger$ We remark that all cases considered in this section actually occur, for a surface $S$ may be constructed to satisfy the requirements of $\S 1$ with values arbitrarily assigned to $1 / R, \psi, p, p^{\prime}$ at a point of $C$.

We state the chief results of this section in

Theorem 2: On a zero ruling of a surface $S$ there is generally one flat point. The geodesic curvature of an isogonal trajectory of the rulings at a point of intersection with a zero ruling, not a flat point, is zero for all trajectories or for none.

Yale University, 1918.

* If two ruled surfaces are applicable the rulings correspond necèssarily unless both surfaces are applicable to the same quadric. See Eisenhart, p. 347.

† Scheffers, p. 146. 